doi:10.13108/2021-13-3-27

\title{
EXPONENTIAL SERIES IN NORMED SPACES OF ANALYTIC FUNCTIONS
}

\author{
R.A. BASHMAKOV, K.P. ISAEV, A.A. MAKHOTA
}

\begin{abstract}
There is a classical well-known theorem by A.F. Leontiev on representing functions analytic in a convex domain $D$ and continuous up to the boundary by series of form $\sum_{k=1}^{\infty} f_{k} e^{\lambda_{k} z}$ converging in the topology of the space $H(D)$, that is, uniformly on compact subsets in $D$.
\end{abstract}

In the paper we prove the possibility of representing the functions in

$$
A_{0}(D)=\left\{f \in H(D) \bigcap C(\bar{D}):\|f\|:=\sup _{z \in \bar{D}}|f(z)|\right\}
$$

by the exponential series converging in a stronger topology, namely, there exists an integer number $s>0$ such that

1) for each bounded convex domain $D$ there exists a system of exponentials $e^{\lambda_{k} z}, k \in \mathbb{N}$, such that each function $f \in H(D) \bigcap C^{(s)}(\bar{D})$ is represented as a series over this system converging in the norm of the space $A_{0}(D)$;

2) for each bounded convex domain $D$ there exists a system of exponentials $e^{\lambda_{k} z}, k \in \mathbb{N}$ such that each function $f \in A_{0}(D)$ is represented as a series over this system converging in the norm

$$
\|f\|=\sup _{z \in D}|f(z)|(d(z))^{s}
$$

where $d(z)$ is the distance from a point $z$ to the boundary of the domain $D$. The number $s$ is related with the existence of entire functions with a maximal possible asymptotic estimate.

In particular cases, when $D$ is a polygon or a domina with a smooth boundary possessing a smooth curvature separated from zero, we can assume that $s=4$.

Keywords: analytic function, entire function, Fourier-Laplace transform, interpolation, exponential series.

Mathematics Subject Classification: 30B50, 30D20

\section{INTRODUCTION}

Let $D$ be a bounded convex domain in the complex plane. In this work we consider a problem on representing the functions functions in the space

$$
A_{0}(D)=\left\{f \in H(D) \bigcap C(\bar{D}):\|f\|:=\sup _{z \in \bar{D}}|f(z)|\right\}
$$

by the exponential series

$$
f(z)=\sum_{k=1}^{\infty} f_{k} e^{\lambda_{k} z}, \quad z \in D, \quad f \in A_{0}(D) .
$$

R.A. Bashmakov, K.P. Isaev, A.A. Makhota, Exponential series in normed spaces of analytic FUNCTIONS.

(C) Bashmakov R.A., Isaev K.P., Makhota A.A. 2021.

The research is made in the framework of executing the Developing Program of Scientific and Educational mathematical center of Privolzhsky Federal District (agreement no. 075-02-2021-1393).

Submitted June 8, 2021. 
In the context of this work, the notation $A_{0}(D)$ is more convenient than a traditional one $A(D)$ since we shall consider a parametrized family of normed spaces $A_{n}(D), n \in \mathbb{Z}$. The possibility of such representation is implied by the classical theorem by A.F. Leontiev, see [1, Thm. 5.3.2], but the series in this theorem converge in the topology of the space $H(D)$, that is, uniformly on the compact sets in $D$. We are going to prove that the functions in $A_{0}(D)$ can be represented by exponential series converging to its sums in a topology, which is essentially stronger than the topology of uniform convergence on compact sets but at the same time being weaker than the normed topology of $A_{0}(D)$, see Theorem 3.2 . We shall also obtain the formulae for the coefficients of the series. There just few known examples of the normed spaces, in which the expansions into exponential series are possible, that is, in which there exist a basis of exponentials. This is the space $L_{2}$ on a segment, the Sobolev space on a segment [2] and Smirnov and Bergman spaces on convex polygons [3], [4]. It was proved in works [5] and [6] that the Smirnov and Bergman spaces on convex domains with a smooth boundary contain no exponential bases. The main statement of the present work reads as follows. There exists an integer number $s>0$ such that

1) for each bounded convex domain $D$ there exists a system of exponentials $e^{\lambda_{k} z}, k \in \mathbb{N}$, such that each function $f \in H(D) \bigcap C^{(s)}(\bar{D})$ is represented as a series over this system converging in the norm of the space $A_{0}(D)$;

2) for each bounded convex domain $D$ there exists a system of exponentials $e^{\lambda_{k} z}, k \in \mathbb{N}$, such that each function $f \in A_{0}(D)$ is represented as a series over this system converging in the norm

$$
\|f\|=\sup _{z \in D}|f(z)|(d(z))^{s},
$$

where $d(z)$ is the distance from a point $z$ to the boundary of the domain $D$. The number $s$ is related with existence of entire functions with a best possible asymptotic estimate.

In particular cases, when $D$ is a polygon or a domain with a smooth boundary having a curvature separated from zero we can suppose that $s=4$.

\section{Fourier-Laplace tRANSFORMS}

We introduce a family of normed spaces

$$
A_{n}(D)=\left\{f \in H(D) \bigcap C^{(n)}(\bar{D}):\|f\|:=\max _{k=0, \ldots, n} \sup _{z \in D}\left|f^{(k)}(z)\right|<\infty\right\}, \quad n \in \mathbb{N} .
$$

It is obvious that the continuous embeddings $A_{n} \subset A_{n-1}, n \in \mathbb{N}$, hold true and the differential operator

$$
\mathcal{D}^{n}: f \rightarrow f^{(n)}
$$

acts continuously from $A_{n}$ onto $A_{0}$.

We define extra two auxiliary families of normed spaces of entire functions with a continuous parameter $\alpha \in \mathbb{R}$ :

$$
\begin{aligned}
& \mathcal{P}_{\alpha}(D)=\left\{F \in H(\mathbb{C}):\|F\|:=\sup _{\lambda \in \mathbb{C}}|F(\lambda)| e^{-H_{D}(\lambda)-\alpha \ln (|\lambda|+1)}<\infty\right\}, \\
& \widetilde{\mathcal{P}}_{\alpha}(D)=\left\{F \in H(\mathbb{C}):\|F\|:=\sup _{\lambda \in \mathbb{C}}|F(\lambda)| e^{-H_{D}(\lambda)-\alpha \ln (|\lambda|+1)+2 \ln ^{+} \ln (|\lambda|+1)}<\infty\right\},
\end{aligned}
$$

where

$$
H_{D}(\lambda)=\sup _{z \in D} \operatorname{Re} \lambda z
$$

is the support function of the domain $D$ and $\ln ^{+} a=\max (\ln a, 0)$. The following continuous embeddings are obvious: $\widetilde{\mathcal{P}}_{\alpha} \subset \mathcal{P}_{\alpha} \subset \mathcal{P}_{\beta}$ as $\beta \geqslant \alpha$.

Finally, for $a>0$ we let:

$$
B_{a}(D)=\left\{f \in H(D):\|f\|=\sup _{z \in D}|f(z)|(d(z))^{a}<\infty\right\},
$$

where the symbol $d(z)$ denotes the distance from a point $z$ to the boundary $D$ :

$$
d(z)=\inf _{w \notin D}|z-w|, \quad z \in D .
$$


Lemma 2.1. 1. Let $S$ be a linear continuous functional on $A_{n}(D)$ and $\widehat{S}(\lambda)=S\left(e^{\lambda z}\right)$ be its FourierLaplace transform. Then $\widehat{S}(\lambda)$ is an entire function satisfying the estimate

$$
|\widehat{S}(\lambda)| \leqslant\|S\|_{A_{n}^{*}} e^{H_{D}(\lambda)+n \ln (|\lambda|+1)}, \quad \lambda \in \mathbb{C},
$$

that is, $\widehat{S}(\lambda) \in \mathcal{P}_{n}$ and

$$
\|\widehat{S}\|_{\mathcal{P}_{n}} \leqslant\|S\|_{A_{n}^{*}} .
$$

2. If $F(\lambda) \in \widetilde{\mathcal{P}}_{n-1}$, then $F(\lambda) \equiv \widehat{S}(\lambda), \lambda \in \mathbb{C}$, for some linear continuous functional $S=S_{F}$ on the space $A_{n}(D)$. At that,

$$
\|S\|_{A_{n}^{*}} \leqslant C\|F\|_{\widetilde{\mathcal{P}}_{n-1}} .
$$

The constant $C=C(D, n)$ is independent of the function $F$.

Proof. 1. The fact that the Laplace-Fourier transform is an entire function and the formula

$$
\frac{d}{d \lambda} \widehat{S}(\lambda)=S\left(z e^{\lambda z}\right), \quad \lambda \in \mathbb{C}
$$

holds is well-known and in our case it can be easily proved. The estimate implies immediately from the definition of the norm of the functional

$$
|\widehat{S}(\lambda)| \leqslant\|S\| \cdot\left\|e^{\lambda z}\right\|_{A_{n}(D)}=\|S\| \sup _{k=0, \ldots, n}|\lambda|^{k} \sup _{z \in D} e^{\operatorname{Re} \lambda z} \leqslant\|S\| e^{H_{D}(\lambda)+n \ln (|\lambda|+1)}, \quad \lambda \in \mathbb{C} .
$$

2a. We first consider the case $n=0$. Let $F \in \mathcal{P}_{-1}$ and

$$
\gamma(\zeta)=\sum_{k=0}^{\infty} \frac{F^{(k)}(0)}{\zeta^{k+1}}, \quad \zeta \in \mathbb{C} \backslash \bar{D},
$$

be its Borel transform. As it is known, in the half-plane $\Pi(\varphi)=\left\{\operatorname{Re} \zeta e^{i \varphi}>h_{D}(\varphi)\right\}$, where $h_{D}(\varphi)=$ $r^{-1} H_{D}\left(r e^{i \varphi}\right)$, the identity holds:

$$
\gamma(\zeta)=\int_{0}^{\infty} F(\lambda) e^{-\zeta \lambda} d \lambda
$$

where the integral is taken over the ray $\left\{\lambda=r e^{i \varphi}, r>0\right\}$. Therefore, for $\zeta \in \Pi(\varphi)$ the inequality holds:

$$
|\gamma(\zeta)| \leqslant \sup _{\lambda \in \mathbb{C}}|F(\lambda)| e^{-H_{D}(\lambda)+\ln (|\lambda|+1)+2 \ln ^{+} \ln (|\lambda|+1)} \int_{0}^{\infty} \frac{1}{(r+1) \ln ^{2}(r+1)} d r,
$$

and hence,

$$
|\gamma(\zeta)| \leqslant\|F\|_{\widetilde{\mathcal{P}}_{-1}}, \quad \zeta \in \Pi(\varphi) .
$$

In view of $\mathbb{C} \backslash \bar{D}=\bigcup_{\varphi \in[0 ; 2 \pi)} \Pi(\varphi)$ this implies the boundedness of the function $\gamma$ :

$$
|\gamma(\zeta)| \leqslant\|F\|_{\widetilde{\mathcal{P}}_{-1}}, \quad \zeta \in \mathbb{C} \backslash \bar{D} .
$$

We take an arbitrary function $f \in A_{0}(D)$. Without loss of generality we suppose that $0 \in D$. We take an arbitrary number $t \in(1 ; 2]$ and a closed Jordan contour $C_{t}$ enveloping the domain $D$ and lying in the domain $t D=\{t z: z \in D\}$. The function $f(z)$ is uniformly continuous on the compact set $\bar{D}$ and hence the function

$$
B(t)=\frac{1}{2 \pi i} \int_{C_{t}} \gamma(\zeta) f\left(\frac{\zeta}{t}\right) d \zeta
$$

is uniformly continuous on the interval $(1 ; 2)$; in particular, the limit

$$
<f, \gamma>:=\lim _{t \rightarrow 1} B(t)
$$

is well-defined and it follows from (1) that

$$
|<f, \gamma>| \leqslant \frac{|\partial D|}{2 \pi}\|f\|_{A_{0}}\|F\|_{\widetilde{\mathcal{P}}_{-1}} .
$$


Thus, the formula

$$
S(f)=<f, \gamma>, \quad f \in A_{0},
$$

defines a linear continuous functional on $A_{0}$ and

$$
\|S\|_{A_{0}^{*}} \leqslant \frac{|\partial D|}{2 \pi}\|F\|_{\widetilde{\mathcal{P}}_{-1}}
$$

Moreover, by the Borel formula we have:

$$
S\left(e^{\lambda z}\right)=\lim _{t \rightarrow 1} \frac{1}{2 \pi i} \int_{C_{t}} \gamma(z) e^{\lambda \frac{z}{t}} d z=\lim _{t \rightarrow 1} F\left(\frac{\lambda}{t}\right)=F(\lambda) .
$$

Remark 2.1. By a more lengthy calculations one can prove that $\gamma(z)$ is continuous on $\mathbb{C} \backslash D$ and the functional $S=S_{F}$ is defined by the formula

$$
S_{F}(f)=\frac{1}{2 \pi i} \int_{\partial D} \gamma(\zeta) f(\zeta) d \zeta, \quad f \in A_{0} .
$$

$2 \mathrm{~b}$. We proceed to the case of an arbitrary $n$. It is easy to confirm that the linear operator

$$
L_{n}(F)(\lambda):=\lambda^{-n}\left(F(\lambda)-\sum_{k=0}^{n-1} \frac{F^{(k)}(0)}{k !} \lambda^{k}\right), \quad \lambda \in \mathbb{C},
$$

acts continuously from $\widetilde{\mathcal{P}}_{n-1}$ into $\widetilde{\mathcal{P}}_{-1}$. Let $F \in \widetilde{\mathcal{P}}_{n-1}$. It follows from statement proved in Item $2 \mathrm{a}$ that

$$
F_{n}=L_{n}(F)=\widehat{S}_{n}
$$

for some $S_{n} \in A_{0}^{*}$. Then the formula

$$
S(f)=S_{n}\left(\mathcal{D}^{n} f\right)+\sum_{k=0}^{n-1} F^{(k)}(0) f^{(k)}(0), \quad f \in A_{n},
$$

defines a linear continuous functional on $A_{n}$ and

$$
\begin{aligned}
\|S\|_{A_{n}^{*}} & =\left\|S_{n}\right\| A_{0}^{*}\left\|\mathcal{D}^{n} f\right\|_{A_{0}}+\sum_{k=0}^{n-1}\left\|\delta^{(k)}\right\| \cdot\|F\|_{\widetilde{\mathcal{P}}_{n-1}} \\
& \leqslant \frac{|\partial D|}{2 \pi}\left\|F_{n}\right\|_{\widetilde{\mathcal{P}}_{-1}}\left\|\mathcal{D}^{n} f\right\|_{A_{0}}+\sum_{k=0}^{n-1}\left\|\delta^{(k)}\right\| \cdot\|F\|_{\widetilde{\mathcal{P}}_{n-1}} \\
& \leqslant\left(\frac{|\partial D|}{2 \pi}\left\|L_{n}\right\|\left\|\mathcal{D}^{n} f\right\|_{A_{0}}+\sum_{k=0}^{n-1}\left\|\delta^{(k)}\right\|\right)\|F\|_{\widetilde{\mathcal{P}}_{n-1}},
\end{aligned}
$$

where by $\left\|\delta^{(k)}\right\|$ we denote the norm of the functional $F \rightarrow F^{(k)}(0)$ in the space $\mathcal{P}_{n-1}$.

The identity $\widehat{S}=F$ is checked by straightforward substitution.

Corollary 2.1. Each function $f \in A_{n}(D)$ is the Fourier-Laplace transform of some functional $S$ on the space $\widetilde{\mathcal{P}}_{n-1}$.

Proof. For $n=0$ the form $\langle f, \gamma\rangle$ defined in (2) is bilinear on $A_{0} \times \mathcal{P}_{-1}$ and for a fixed $f$ it is a linear functional $S_{f}$ on $\mathcal{P}_{-1}$. Estimate $(3)$ implies the continuity of this functional. The identity $\widehat{S}_{f}=f$ is implied by the Cauchy formula. For an arbitrary $n$ the proof also follows from Item $2 \mathrm{~b}$.

\section{Interpolation in SPACES $\widetilde{\mathcal{P}}_{\alpha}$}

The properties of the systems of exponentials $e^{\lambda_{k} z}, k \in \mathbb{N}$, are traditionally described by a characteristic entire function $L(\lambda)$ with zeroes $\lambda_{k}, k \in \mathbb{N}$. In this paper we employ the results of works [7]. In particular, Theorem 1 in this work implies easily the following theorem. 
Theorem A. There exists an universal constant $A$ such that for each bounded convex domain $D$ there exists an entire function $f(\lambda)$ possessing the properties:

1) the set of zeroes $\lambda_{k}, k \in \mathbb{N}$, is separated in the sense that for some $\delta \in(0 ; 1)$ the circles $B_{\delta}\left(\lambda_{k}\right)=$ $B\left(\lambda_{k}, \delta(|\lambda|+1)^{-1}\right)$ are mutually disjoint;

2) the relations hold:

$$
\begin{aligned}
& |\ln | f(\lambda)\left|-H_{D}(\lambda)\right| \leqslant A \ln (|\lambda|+1)+C, \quad \lambda \notin \bigcup_{k \in \mathbb{N}} B_{\delta}\left(\lambda_{k}\right), \\
& |\ln | f^{\prime}\left(\lambda_{k}\right)\left|-H_{D}\left(\lambda_{k}\right)\right| \leqslant A \ln \left(\left|\lambda_{k}\right|+1\right)+C^{\prime}, \quad k \in \mathbb{N},
\end{aligned}
$$

where $C$ and $C^{\prime}$ are constants independent of the domain $D$.

By using this theorem, we are going to show the existence of two entire functions $L_{ \pm}$, which will be employed as a tool for constructing the systems of exponentials.

Theorem 3.1. There exists universal constants $b>0$ and $q \in(1 ; 2]$ such that for each bounded convex domain $D$ there exist entire functions $L_{+}(\lambda)$ and $L_{-}(\lambda)$ possessing the following properties: $1)$ the sets of zeroes $\Lambda_{+}$and $\Lambda_{-}$of these functions are separated in the sense that for some $\delta \in(0 ; 1)$ the circles $B_{\delta}(\lambda)=B\left(\lambda, \delta(|\lambda|+1 \mid)^{-1}\right), \lambda \in \Lambda_{+}\left(\lambda \in \Lambda_{-}\right)$are mutually disjoint;

2) the function $L_{+}$satisfies the relations

$$
\begin{aligned}
& H_{D}(\lambda)+q \ln (|\lambda|+1) \leqslant \ln \left|L_{+}(\lambda)\right| \leqslant H_{D}(\lambda)+b \ln (|\lambda|+1)+C, \quad \lambda \notin \bigcup_{w \in \Lambda_{+}} B_{\delta}(w), \\
& \ln \left|L_{+}^{\prime}(\lambda)\right| \geqslant H_{D}(\lambda)+q \ln (|\lambda|+1), \quad \lambda \in \Lambda_{+} ;
\end{aligned}
$$

3) the function $L_{-}$satisfies the relations

$$
\begin{aligned}
& H_{D}(\lambda)-b \ln (|\lambda|+1)+C \leqslant \ln \left|L_{-}(\lambda)\right| \leqslant H_{D}(\lambda)-q \ln (|\lambda|+1), \quad \lambda \notin \bigcup_{w \in \Lambda_{-}} B_{\delta}(w), \\
& \ln \left|L_{-}^{\prime}(\lambda)\right| \geqslant H_{D}(\lambda)-b \ln (|\lambda|+1), \quad \lambda \in \Lambda_{-} .
\end{aligned}
$$

Proof. Let $f$ be a function, the existence of which is stated in Theorem A. We take a polynomial $P(\lambda)$ of degree $[A]+2$ so that the set of the zeroes of the function $L_{+}=f P$ is separated. The desired estimates for $L_{+}$are implied by the estimates in Theorem $\mathrm{A}$ in a trivial way for $b=[A]+2+A$, $q=[A]+2-A$.

Let $N=[A]+2$ and

$$
Q(\lambda)=\prod_{k=1}^{N}\left(\lambda-\lambda_{k}\right)
$$

where $\left\{\lambda_{k}, k=1,2, \ldots, N\right\}$ are the first $N$ zeroes of the function $f$ from Theorem A taking in the ascending order of their absolute values. Then the function $L_{-}=\frac{f}{Q}$ satisfies the desired estimates.

Lemma 3.1. For each function $F \in \widetilde{\mathcal{P}}_{\alpha}, \alpha \leqslant q$ its Lagrangue series by the function $L_{+}$converges to the function $F$ uniformly on compact sets and moreover, as $\alpha \leqslant q-1$, this series converges in the norm of the space $\mathcal{P}_{b}$.

Proof. The separated property of the zero set of the function $L_{+}$implies the existence of the system of curves $\Gamma_{m}$ not intersecting with the set $\bigcup_{k=1}^{\infty} B_{\delta}\left(\lambda_{k}\right)$ and satisfying the estimate

$$
\min _{z \in \Gamma_{m}}|z| \rightarrow \infty, \quad\left|\Gamma_{m}\right|=O\left(\min _{z \in \Gamma_{m}}|z|\right), \quad m \rightarrow \infty .
$$

By the lower bound for $L_{+}$in Theorem 3.1 , for each function $F \in \widetilde{\mathcal{P}}_{\alpha}, \alpha \leqslant q$, we have:

$$
\left|\frac{1}{2 \pi i} \int_{\gamma_{m}} \frac{F(z) d z}{(z-\lambda) L_{+}(z)}\right|=O\left(\ln ^{-2}\left(\min _{z \in \Gamma_{m}}|z|+1\right)\right)=o(1), \quad m \rightarrow \infty .
$$


Therefore, the Lagrange series

$$
F(\lambda)=\sum_{k=1}^{\infty} F\left(\lambda_{k}\right) \frac{L_{+}(\lambda)}{\left(\lambda-\lambda_{k}\right) L_{+}^{\prime}\left(\lambda_{k}\right)}, \quad \lambda \in \mathbb{C},
$$

converges uniformly on compact sets.

The upper bounds for $\left|L_{+}\right|$in Theorem 3.1 and the Lipschitz property of the function $H_{D}(\lambda)+$ $b \ln (|\lambda|+1)$ we obtain an uniform in $k$ estimate:

$$
\left\|\frac{L_{+}(\lambda)}{\left(\lambda-\lambda_{k}\right) L_{+}^{\prime}\left(\lambda_{k}\right)}\right\|_{\mathcal{P}_{b}} \leqslant \text { Const } \cdot \frac{1}{\left|L_{+}^{\prime}\left(\lambda_{k}\right)\right|}, \quad k \in \mathbb{N}
$$

and therefore,

$$
\left\|\sum_{k \geqslant N} F\left(\lambda_{k}\right) \frac{L_{+}(\lambda)}{\left(\lambda-\lambda_{k}\right) L_{+}^{\prime}\left(\lambda_{k}\right)}\right\|_{\mathcal{P}_{b}} \leqslant \text { Const } \cdot \sum_{k \geqslant N} \frac{\left|F\left(\lambda_{k}\right)\right|}{\left|L_{+}^{\prime}\left(\lambda_{k}\right)\right|} .
$$

Since $\lambda_{k}$ are the zeroes of an entire function of exponential type, then for $F \in \widetilde{\mathcal{P}}_{\alpha}, \alpha \leqslant q-1$ we have:

$$
\left\|\sum_{k \geqslant N} F\left(\lambda_{k}\right) \frac{L_{+}(\lambda)}{\left(\lambda-\lambda_{k}\right) L_{+}^{\prime}\left(\lambda_{k}\right)}\right\|_{\mathcal{P}_{b}} \leqslant \text { Const } \cdot \sum_{k \geqslant N} \frac{\left(\left|\lambda_{k}\right|+1\right)^{\alpha-q}}{\ln ^{2}\left(\left|\lambda_{k}\right|+1\right)} \rightarrow 0, \quad N \rightarrow \infty .
$$

Lemma 3.2. For each function $F \in \widetilde{\mathcal{P}}_{\alpha}, \alpha \leqslant-b$, its Lagrange series by the function $L_{-}$converges to the function $F$ uniformly on compact sets and moreover, as $\alpha \leqslant-b-1$, this series diverges in the norm of the space $\mathcal{P}_{-q}$.

Proof. By the separated property of the zeroes of the function $L_{-}$and by the lower bounds for $\left|L_{-}\right|$ we get:

$$
\left|\frac{1}{2 \pi i} \int_{\gamma_{m}} \frac{F(z) d z}{(z-\lambda) L_{-}(z)}\right|=O\left(\ln ^{-2}\left(\min _{z \in \Gamma_{m}}|z|+1\right)\right)=o(1), \quad m \rightarrow \infty .
$$

Therefore, the Lagrange series

$$
F(\lambda)=\sum_{k=1}^{\infty} F\left(\lambda_{k}\right) \frac{L_{-}(\lambda)}{\left(\lambda-\lambda_{k}\right) L_{-}^{\prime}\left(\lambda_{k}\right)}, \quad \lambda \in \mathbb{C},
$$

converges uniformly on compact sets.

By the lower bounds for $\left|L_{-}\right|$in Theorem 3.1 and the Lipschitz property of the function $H_{D}(\lambda)+$ $b \ln (|\lambda|+1)$ we get a uniform in $k$ estimate

$$
\left\|\frac{L_{-}(\lambda)}{\left(\lambda-\lambda_{k}\right) L_{-}^{\prime}\left(\lambda_{k}\right)}\right\|_{\mathcal{P}_{-q}} \leqslant \text { Const } \cdot \frac{1}{\left|L_{+}^{\prime}\left(\lambda_{k}\right)\right|}, \quad k \in \mathbb{N},
$$

and hence,

$$
\left\|\sum_{k \geqslant N} F\left(\lambda_{k}\right) \frac{L_{-}(\lambda)}{\left(\lambda-\lambda_{k}\right) L_{-}^{\prime}\left(\lambda_{k}\right)}\right\|_{\mathcal{P}_{-q}} \leqslant \text { Const } \cdot \sum_{k \geqslant N} \frac{\left|F\left(\lambda_{k}\right)\right|}{\left|L_{-}^{\prime}\left(\lambda_{k}\right)\right|} .
$$

Since $\lambda_{k}$ are the zeroes of an entire function of exponential type, then for $F \in \widetilde{\mathcal{P}}_{\alpha}, \alpha \leqslant-b-1$ we have:

$$
\left\|\sum_{k \geqslant N} F\left(\lambda_{k}\right) \frac{L_{+}(\lambda)}{\left(\lambda-\lambda_{k}\right) L_{+}^{\prime}\left(\lambda_{k}\right)}\right\|_{\mathcal{P}_{b_{+}}} \leqslant \text {Const } \cdot \sum_{k \geqslant N} \frac{\left(\left|\lambda_{k}\right|+1\right)^{\alpha+b}}{\ln ^{2}\left(\left|\lambda_{k}\right|+1\right)} \rightarrow 0, \quad N \rightarrow \infty .
$$

We let $s=[b]+2=2([\mathrm{~A}]+2)$. According to Corollary of Lemma 2.1, each function in the space $A_{s}(D)$ is the Fourier-Laplace transform of some functional on the space $\mathcal{\mathcal { P }}_{s-1}$. Since the family of spaces $\mathcal{P}_{\alpha}$ is continuously embedded, each function $f \in A_{s}(D)$ is the Fourier-Laplace transform of some functional on the space $\mathcal{P}_{b}$. 
Theorem 3.2. Each function $f \in A_{s}$ can be represented as the series

$$
f(z)=\sum_{k=1}^{\infty} f_{k} e^{\lambda_{k} z}, \quad z \in D,
$$

converging in uniform norm over $\bar{D}$. The coefficients can be found by the formulae

$$
f_{k}=S\left(\frac{L_{+}(\lambda)}{\left(\lambda-\lambda_{k}\right) L_{+}^{\prime}\left(\lambda_{k}\right)}\right), \quad k \in \mathbb{N},
$$

where $S=S_{f}$ is a functional on the space $\mathcal{P}_{b}$ generated by the function $f$.

Proof. We take an arbitrary point $z \in \bar{D}$. The function $e^{\lambda z}, \lambda \in \mathbb{C}$, belongs to all spaces $\mathcal{P}_{\alpha}, \alpha \geqslant 0$, and by Lemma 3.1 , the Lagrange series

$$
e^{\lambda z}=\sum_{k=1}^{\infty} e^{\lambda_{k} z} \frac{L_{+}(\lambda)}{\left(\lambda-\lambda_{k}\right) L_{+}^{\prime}\left(\lambda_{k}\right)}, \quad \lambda \in \mathbb{C},
$$

converges in the space $\mathcal{P}_{b}$. Therefore, a point-wise identity holds:

$$
f(z)=S\left(e^{\lambda z}\right)=\sum_{k=1}^{\infty} e^{\lambda_{k} z} S\left(\frac{L_{+}(\lambda)}{\left(\lambda-\lambda_{k}\right) L_{+}^{\prime}\left(\lambda_{k}\right)}\right)=\sum_{k=1}^{\infty} f_{k} e^{\lambda_{k} z} .
$$

We are going to prove a uniform on $\bar{D}$ convergence of this series. By estimate (4) we have:

$$
\left|f_{k}\right| \leqslant\left\|S_{f}\right\|\left\|\frac{L_{+}(\lambda)}{\left(\lambda-\lambda_{k}\right) L_{+}^{\prime}\left(\lambda_{k}\right)}\right\|_{\mathcal{P}_{b}} \leqslant \text { Const } \cdot \frac{1}{\left|L_{+}^{\prime}\left(\lambda_{k}\right)\right|}, \quad k \in \mathbb{N} .
$$

Hence,

$$
\left\|\sum_{k \geqslant N} e^{\lambda_{k} z} \frac{L_{+}(\lambda)}{\left(\lambda-\lambda_{k}\right) L_{+}^{\prime}\left(\lambda_{k}\right)}\right\| \leqslant \text { Const } \cdot \sum_{k \geqslant N} \frac{e^{H_{D}\left(\lambda_{k}\right)}}{L_{+}^{\prime}\left(\lambda_{k}\right)} .
$$

By the lower bounds for the derivatives and the inequality $q>1$ this implies:

$$
\left\|\sum_{k \geqslant N} e^{\lambda_{k} z} \frac{L_{+}(\lambda)}{\left(\lambda-\lambda_{k}\right) L_{+}^{\prime}\left(\lambda_{k}\right)}\right\| \leqslant \text { Const } \cdot \sum_{k \geqslant N} \frac{1}{\left(\left|\lambda_{k}\right|+1\right)^{q}} \rightarrow 0, \quad N \rightarrow \infty .
$$

By Corollary of Lemma 2.1, each function in $A_{0}$ is the Fourier-Laplace transform of some functional $S=S_{f}$ on $\widetilde{\mathcal{P}}_{-1}$. Since the spaces $\mathcal{P}_{\alpha}$ are continuously embedded, the functional $S_{f}$ acts also in the spaces $\mathcal{P}_{-q}$ for $q<-1$.

Theorem 3.3. Each function $f \in A_{0}$ can be represented by the series

$$
f(z)=\sum_{k=1}^{\infty} f_{k} e^{\lambda_{k} z}, \quad z \in D,
$$

converging in the space $B_{s}(D)$, where $s=[b]+2$. The coefficients can be calculated by the formulae:

$$
f_{k}=S\left(\frac{L_{-}(\lambda)}{\left(\lambda-\lambda_{k}\right) L_{-}^{\prime}\left(\lambda_{k}\right)}\right), \quad k \in \mathbb{N}
$$

where $S=S_{f}$ is a functional on the space $\mathcal{P}_{-q}$ generated by the function $f$.

Proof. We take an arbitrary point $z \in D$. The function $e^{\lambda z}, \lambda \in \mathbb{C}, z \in D$, belongs to all spaces $\mathcal{P}_{\alpha}$ and by Lemma 3.1 the Lagrange series

$$
e^{\lambda z}=\sum_{k=1}^{\infty} e^{\lambda_{k} z} \frac{L_{-}(\lambda)}{\left(\lambda-\lambda_{k}\right) L_{-}^{\prime}\left(\lambda_{k}\right)}, \quad \lambda \in \mathbb{C},
$$


converges in the space $\mathcal{P}_{-q}$. Therefore, a pointwise identity holds:

$$
f(z)=S\left(e^{\lambda z}\right)=\sum_{k=1}^{\infty} e^{\lambda_{k} z} S\left(\frac{L_{-}(\lambda)}{\left(\lambda-\lambda_{k}\right) L_{-}^{\prime}\left(\lambda_{k}\right)}\right)=\sum_{k=1}^{\infty} f_{k} e^{\lambda_{k} z} .
$$

Let us prove the convergence of this series in the norm of the space $B_{s}(D)$. By estimate (5) we have:

$$
\left|f_{k}\right| \leqslant\left\|S_{f}\right\|\left\|\frac{L_{-}(\lambda)}{\left(\lambda-\lambda_{k}\right) L_{-}^{\prime}\left(\lambda_{k}\right)}\right\|_{\mathcal{P}_{-q}} \leqslant \text { Const } \cdot \frac{1}{\left|L_{-}^{\prime}\left(\lambda_{k}\right)\right|}, \quad k \in \mathbb{N} .
$$

Therefore,

$$
\left\|\sum_{k \geqslant N} e^{\lambda_{k} z} \frac{L_{-}(\lambda)}{\left(\lambda-\lambda_{k}\right) L_{-}^{\prime}\left(\lambda_{k}\right)}\right\|_{B_{s}} \leqslant \text { Const } \cdot \sum_{k \geqslant N} \frac{\left\|e^{\lambda_{k} z}\right\|_{B_{s}}}{L_{-}^{\prime}\left(\lambda_{k}\right)} .
$$

To estimate the norms of the exponentials in the space $B_{s}(D)$ we make use of the inequality

$$
H_{D}(\lambda)-\operatorname{Re} \lambda z=\sup _{\zeta \in D} \operatorname{Re} \lambda(\zeta-z) \geqslant \sup _{|\zeta-z|<d(z)} \operatorname{Re} \lambda(\zeta-z)=d(z)|\lambda|
$$

where $z \in D, \lambda \in \mathbb{C}, d(z)$ is the distance from a point $z \in D$ to the boundary $D$. Hence, for sufficiently large $|\lambda|$, the inequality holds:

$$
\left\|e^{\lambda z}\right\|_{B_{s}}=e^{H_{D}(\lambda)} e^{\sup _{z \in D}(-d(z)|\lambda|+s \ln d(z))} \leqslant\left(\frac{s}{e}\right)^{s} e^{H_{D}(\lambda)-s \ln |\lambda|} .
$$

Therefore, by the lower bounds for the derivatives and the relations $b-s=b-([b]+2)=-q<-1$ we get:

$$
\left\|\sum_{k \geqslant N} e^{\lambda_{k} z} \frac{L_{-}(\lambda)}{\left(\lambda-\lambda_{k}\right) L_{-}^{\prime}\left(\lambda_{k}\right)}\right\| \leqslant \text { Const } \cdot \sum_{k \geqslant N} \frac{1}{\left(\left|\lambda_{k}\right|+1\right)^{q}} \rightarrow 0, \quad N \rightarrow \infty .
$$

In conclusion we mention that Theorems 3.2 and 3.3 imply the following statement.

Theorem 3.4. If for a support function $H_{D}(\lambda)$ of a bounded convex domain $D$ there exists an entire function $L$ obeying the conditions:

1) the set of zeroes $\lambda_{k}, k \in \mathbb{N}$, is separated in the sense that for some $\delta \in(0 ; 1)$ the circles $B_{\delta}\left(\lambda_{k}\right)=$ $B\left(\lambda_{k}, \delta(|\lambda|+1)^{-1}\right)$ are mutually disjoint;

2) the relations hold:

$$
\begin{aligned}
& |\ln | L(\lambda)\left|-H_{D}(\lambda)\right| \leqslant A \ln (|\lambda|+1)+C, \quad \lambda \notin \bigcup_{k=1}^{\infty} B_{\delta}\left(\lambda_{k}\right), \\
& |\ln | L^{\prime}\left(\lambda_{k}\right)\left|-H_{D}\left(\lambda_{k}\right)\right| \leqslant A \ln \left(\left|\lambda_{k}\right|+1\right)+C^{\prime}, \quad k \in \mathbb{N},
\end{aligned}
$$

and $s=2([A]+2)$, then

1. There exists a system of exponentials $e^{\lambda_{k} z}, k \in \mathbb{N}$, such that each function $f \in A_{s}$ is represented by the series

$$
f(z)=\sum_{k=1}^{\infty} f_{k} e^{\lambda_{k} z}, \quad z \in D
$$

converging in the uniform norm over $\bar{D}$.

2. There exists a system of exponentials $e^{\lambda_{k} z}, k \in \mathbb{N}$, such that each function $f \in A_{0}$ is represented by a series

$$
f(z)=\sum_{k=1}^{\infty} f_{k} e^{\lambda_{k} z}, \quad z \in D
$$

converging in the norm

$$
\|f\|=\sup _{z \in D}|f(z)|(d(z))^{s}
$$


It was shown in work [3] that for bounded convex polygons there exist entire functions $L$ with constant $A=0$. In work [8], for the domains $D$, the boundary of which has a continuous curvature separated from zero, there were constructed entire functions $L$ with constant $A=\frac{1}{2}$. Thus, for these classes of domains, we can suppose that $s=4$ in Theorems 3.23 .4

\section{BIBLIOGRAPHY}

1. A.F. Leontiev. Exponential series. Nauka, Moscow (1976). (in Russian).

2. D.L. Russell. On exponential bases for the Sobolev spaces over an interval // J. Math. Anal. Appl. 87:2, 528-550 (1982).

3. B.Ya. Levin, Yu.I. Lyubarskii. Interpolation by means of special classes of entire functions and related expansions in series of exponentials // Izv. AN SSSR. Ser. Matem. 39:3, 657-702 (1975). [Math. USSR-Izv. 9:3, 621-662 (1975).]

4. K.P. Isaev. Riesz bases of exponents in Bergman spaces on convex polygons // Ufimskij Matem. Zhurn. 2:1, 71-86 (2010). (in Russian).

5. V.I. Lutsenko. Unconditional bases of exponentials in Smirnov spaces // PhD thesis. Institute of Mathematics, Ufa Scientific Center, RAS (1992). (in Russian).

6. K.P. Isaev, R.S. Yulmukhametov. The absence of unconditional bases of exponentials in Bergman spaces on non-polygonal domains // Izv. RAN. Set. Matem. 71:6, 69-90 (2007). [Izv. Math. 71:6, $1145-1166$ (2007).]

7. K.P. Isaev. On entire functions with given asymptotic behavior // Probl. Anal. Issues Anal. 7(25):2, 12-30 (2018).

8. Yu.I. Lyubarskii. Exponential series in Smirnov spaces and interpolation by entire functions of special classes // Izv. AN SSSR. Ser. Matem. 52:3, 559-580 (1988). [Math. USSR-Izv. 32:3, 563586 (1989).]

Rustem Abdraufovich Basmakov,

Bashkir State University,

Zaki Validi str. 32,

450074, Ufa, Russia

E-mail: bashmakov_rustem@mail.ru

Konstatin Petrovich Isaev, Institute of Mathematics,

Ufa Federal Research Center, RAS,

Chernyshebvsky str. 112,

450008, Ufa, Russia

E-mail: orbit81@list.ru

Alla Alexandrovna Makhota,

Bashkir State University,

Zaki Validi str. 32,

450074, Ufa, Russia

E-mail: allarum@mail.ru 